\title{
Inhibition of Haemonchus contortus larval development by fungal lectins
}

\author{
Christian Heim ${ }^{1}$, Hubertus Hertzberg ${ }^{1}$, Alex Butschi ${ }^{2}$, Silvia Bleuler-Martinez ${ }^{3}$, Markus Aebi ${ }^{3}$, Peter Deplazes ${ }^{1}$,
} Markus Künzler ${ }^{3}$ and Saša Štefanić ${ }^{* *}$

\begin{abstract}
Background: Lectins are carbohydrate-binding proteins that are involved in fundamental intra- and extracellular biological processes. They occur ubiquitously in nature and are especially abundant in plants and fungi. It has been well established that certain higher fungi produce lectins in their fruiting bodies and/or sclerotia as a part of their natural resistance against free-living fungivorous nematodes and other pests. Despite relatively high diversity of the glycan structures in nature, many of the glycans targeted by fungal lectins are conserved among organisms of the same taxon and sometimes even among different taxa. Such conservation of glycans between free-living and parasitic nematodes is providing us with a useful tool for discovery of novel chemotherapeutic and vaccine targets. In our study, a subset of fungal lectins emanating from toxicity screens on Caenorhabditis elegans was tested for their potential to inhibit larval development of Haemonchus contortus.
\end{abstract}

Methods: The effect of Coprinopsis cinerea lectins - CCL2, CGL2, CGL3; Aleuria aurantia lectin - AAL; Marasmius oreades agglutinin - MOA; and Laccaria bicolor lectin - Lb-Tec2, on cultivated Haemonchus contortus larval stages was investigated using a larval development test (LDT). To validate the results of the toxicity assay and determine lectin binding capacity to the nematode digestive tract, biotinylated versions of lectins were fed to pre-infective larval stages of H. contortus and visualized by fluorescent microscopy. Lectin histochemistry on fixed adult worms was performed to investigate the presence and localisation of lectin binding sites in the disease-relevant developmental stage.

Results: Using an improved larval development test we found that four of the six tested lectins: AAL, CCL2, MOA and CGL2, exhibited a dose-dependent toxicity in LDT, as measured by the number of larvae developing to the L3 stage. In the case of AAL, CGL2 and MOA lectin, doses as low as $5 \mu \mathrm{g} / \mathrm{ml}$ caused $>95 \%$ inhibition of larval development while $40 \mathrm{\mu g} / \mathrm{ml}$ were needed to achieve the same inhibition by CCL2 lectin. MOA was the only lectin tested that caused larval death while other toxic lectins had larvistatic effect manifesting as L1 growth arrest. Using lectin histochemistry we demonstrate that of all lectins tested, only the four toxic ones displayed binding to the larvae's gut and likewise were found to interact with glycans localized to the gastrodermal tissue of adults.

Conclusion: The results of our study suggest a correlation between the presence of target glycans of lectins in the digestive tract and the lectin-mediated toxicity in Haemonchus contortus. We demonstrate that binding to the structurally conserved glycan structures found in $\mathrm{H}$. contortus gastrodermal tissue by the set of fungal lectins has detrimental effect on larval development. Some of these glycan structures might represent antigens which are not exposed to the host immune system (hidden antigens) and thus have a potential for vaccine or drug development. Nematotoxic fungal lectins prove to be a useful tool to identify such targets in parasitic nematodes.

Keywords: Haemonchus contortus, Fungal lectins, Nematotoxicity, Glycan targets, Vaccine development, Larval development test (LDT)

\footnotetext{
* Correspondence: sasa.stefanic@uzh.ch

'Institute of Parasitology, University of Zurich, Winterthurerstrasse 266a, 8057

Zurich, Switzerland

Full list of author information is available at the end of the article
}

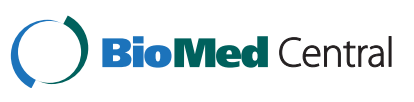

(c) 2015 Heim et al. Open Access This article is distributed under the terms of the Creative Commons Attribution 4.0 International License (http://creativecommons.org/licenses/by/4.0/), which permits unrestricted use, distribution, and reproduction in any medium, provided you give appropriate credit to the original author(s) and the source, provide a link to the Creative Commons license, and indicate if changes were made. The Creative Commons Public Domain Dedication waiver (http://creativecommons.org/publicdomain/zero/1.0/) applies to the data made available in this article, unless otherwise stated. 


\section{Background}

At a global perspective, Haemonchus contortus (also known as the barber pole worm) is regarded as the major helminth pathogen of small ruminants causing extensive economic losses in sheep and goat production. The control of $H$. contortus mainly relies on the use of anthelmintics, but widespread resistance to commonly used drugs [1-5] urges the development of new chemotherapeutics and alternative control strategies such as vaccination. Research on vaccines against $H$. contortus identified a range of parasite molecules that conferred high levels of protection against challenge infection [6-10] (reviewed in [11, 12]), mostly induced by antigens of native origin $[13,14]$. Recombinantly expressed analogues generally stimulate lower levels of protection than their native counterparts and inappropriate glycosylation is considered to be the most likely explanation [15]. In fact, some of the most promising vaccine candidates characterized to date, including larval antigen Hc-sL3, and adult antigens H11, H-gal-GP, contain glycan modifications that might be greatly contributing to protective immunity against $H$. contortus. $\mathrm{H} 11$ and $\mathrm{H}$-gal-GP are believed to be components of the hemoglobinase complex in the $H$. contortus adult gut responsible for digestion of the ingested host hemoglobin and are regarded as hidden antigens not exposed to the host immune system $[9,16,17]$.

Attempts were made to further characterize protective immune responses induced by $H$. contortus infection by analyzing sera of vaccinated animals on glycan microarrays in order to discover reactive glycan epitopes which might be involved in protection [18]. This approach led to the identification of novel glycan structures of $H$. contortus that are also known as natural targets of nematotoxic lectins expressed in fungal fruiting bodies [19].
Fungi use such lectins as part of their innate defence against predatory nematodes and other pests [20] and it has been hypothesized that the glycan epitopes of these lectins are often conserved between different species of the same taxon or even across different taxa [21]. Several target glycan structures of fungal defence lectins have been identified in the model nematode Caenorhabditis elegans [19, 22-25]. Binding of the lectins to these glycans as part of surface glycoproteins or glycolipids on the intestinal epithelium led to inhibition of larval development or sometimes even to larval death. Some of these glycans are likely to be conserved in parasitic nematode species like $H$. contortus where they may, based on their hidden nature and their immunogenicity (see above), represent ideal carbohydrate vaccine candidates. Indeed, glycans of $H$. contortus have been identified which are structurally identical with those in C. elegans [26-28] and some of them, like fucosylated N-glycan cores and Gal 1 3GalNAc play a role in acquired immunity against $H$. contortus $[18,27,29]$. Thus, nematotoxic lectins with specificites to such conserved glycan structures may provide us with a tool to identify novel potential vaccine candidates in $H$. contortus.

In order to further validate this approach of carbohydrate vaccine identification, we implemented an in vitro larval development test to investigate anthelmintic properties of six fungal fruiting body lectins (Table 1; Coprinopsis cinerea lectins - CCL2, CGL2, CGL3; Aleuria aurantia lectin - AAL; Marasmius oreades agglutinin MOA; Laccaria bicolor lectin - Lb-Tec2, referred to as Tectonin throughout the text), and used histochemistry on cryosectioned larval and adult stages of $H$. contortus to localize the lectin binding sites. Results of this study identified four fungal lectins with inhibitory activity on

Table 1 Overview of the fungal lectins used for biotoxicity assays in this study

\begin{tabular}{|c|c|c|c|c|c|c|}
\hline Lectin & Origin & Lectin family & $\begin{array}{l}\text { Molecular } \\
\text { Weight }\end{array}$ & Carbohydrate specificity & References & $\begin{array}{l}\text { GenBank accession } \\
\text { number }\end{array}$ \\
\hline \multirow[t]{2}{*}{ CCL2 } & Coprinopsis cinerea & $\beta$-trefoil & 15 & GlcNAc- $\beta 1,4-(F u c-\alpha 1,3-)$ GlcNAc & Schubert et al. [23] & ACD88750 \\
\hline & & & & & Stutz et al. [30] & \\
\hline \multirow[t]{3}{*}{ CGL2 } & Coprinopsis cinerea & Galectin & 16.7 & Galß1-4Glc (lactose) & Walser et al. [31] & AAF34731 \\
\hline & & & & Galß1-4GlcNAc & Butschi et al. [22] & AAF34732 \\
\hline & & & & Galß1-4Fuc & & \\
\hline \multirow[t]{2}{*}{ CGL3 } & Coprinopsis cinerea & Galectin-like & 19 & GlcNAc $\beta 1-4 G \mid c N A c$ & Walti et al. [32] & ABD64675 \\
\hline & & & & GalNAcß1-4GlcNAc & & \\
\hline \multirow[t]{2}{*}{ AAL } & Aleuria aurantia & $\beta$-propeller & 33.4 & Fucose & Fujihashi et al. [33] & BAA12871 \\
\hline & & & & & Wimmerova et al. [34] & \\
\hline \multirow[t]{2}{*}{ MOA } & Marasmius oreades & $\beta$-trefoil (B-type) & 33 & Gala1,3Gal/GalNAc & Wohlschlager et al. [19] & AAL47680 \\
\hline & & & & & Cordara et al. [35] & \\
\hline \multirow[t]{2}{*}{ Tec2 } & Laccaria bicolor & $\beta$-propeller & 23.8 & 2-O-Me-Fucose & Wohlschlager et al. [25] & EDR12168 \\
\hline & & & & 3-O-Me-Mannose & & \\
\hline
\end{tabular}


development of $H$. contortus larval stages (AAL, MOA, CCL2 and CGL2). All toxic lectins displayed specificity for glycan structures present in the digestive tract of the larvae, as well as on the resorptive surface of the adult parasite gut. The results of this study show that not only the structure, but also the location and the function of target glycans of fungal nematotoxic lectins are conserved between $C$. elegans and $H$. contortus.

\section{Methods}

\section{Ethics statement}

The animal experiments with sheep (Ovis aries) were carried out in accordance with Swiss legislation on animal protection (Animal Wellfare Act: TSchG 455) following ethical principles and guidelines for experiments on animals of the Swiss Academy of Sciences and using the protocols approved by the Cantonal Veterinary Office.

\section{Egg isolation and cultivation of Haemonchus contortus larval stages}

Faeces from sheep mono-infected with a pure Haemonchus contortus isolate were used. Depending on the faecal egg count, 1 to $5 \mathrm{~g}$ of faeces were homogenised in $200 \mathrm{ml}$ of tap water until all faecal pellets were broken up and completely dissolved. The eggs were cleaned from coarse particles by passing through a household sieve overlaid with 3 layers of cotton gauze and the flow-through was then filtered through a stack of sieves having $200 \mu \mathrm{m}, 150 \mu \mathrm{m}, 100 \mu \mathrm{m}, 50 \mu \mathrm{m}$ and $32 \mu \mathrm{m}$ mesh diameter. Eggs from the $32 \mu \mathrm{m}$ sieve were transferred into $50 \mathrm{ml}$ Falcon tube and pelleted at $600 \mathrm{~g}$ for $2 \mathrm{~min}$. One $\mathrm{ml}$ of the egg suspension was loaded on the top of the Percoll gradient (GE Healthcare Biosciences AB, SE75184 Uppsala) consisting of $2.5 \mathrm{ml}$ layer of each $45 \%$, $40 \%, 35 \%$ and $30 \%$ (from the bottom to the top) in a $15 \mathrm{ml}$ tube (Sarstedt D-51588 Nuremberg). The gradient was then centrifuged at $1400 \mathrm{~g}$ for $15 \mathrm{~min}$ and eggs were recovered from the middle of the $35 \%$ Percoll fraction. The eggs were subsequently washed four times with double-concentrated (50 x dilution of stock) antibioticantimycotic solution for $10 \mathrm{~min}$ each $\left(\mathrm{Gibco}^{\circ} /\right.$ LifeTechnologies). The egg suspension volume was adjusted with distilled water to obtain a final concentration of 5 eggs per $\mu \mathrm{l}$.

The cultivation medium for hatched larvae contained yeast extract (Becton Dickinson \& Co.), Earle's salts (Sigma), and heat killed and lyophilized bacteria as a food source. One gram of yeast extract was dissolved in $90 \mathrm{ml} 0.85 \% \mathrm{NaCl}$, and then $3 \mathrm{ml}$ of 10x Earle's salt solution per $27 \mathrm{ml}$ yeast extract was added, adjusted to $\mathrm{pH}$ 7.0, sterile filtered and stored frozen in aliquots. Escherichia coli OP 50 strain was cultivated overnight in $50 \mathrm{ml}$ of LB medium at $37^{\circ} \mathrm{C}$. Optical density at $600 \mathrm{~nm}$ wavelength was measured and the cells were pelleted by centrifugation at $2500 \mathrm{~g}$ for $10 \mathrm{~min}$. The bacterial cell pellet was subsequently resolved in the adequate volume of LB medium to obtain $\mathrm{OD}_{600}=15$. The bacterial suspension was heat treated at $65{ }^{\circ} \mathrm{C}$ in a water bath for $5 \mathrm{~min}$, and aliquots of $0.5 \mathrm{ml}$ were frozen in liquid nitrogen for lyophilisation. The lyophilised bacteria were stored at $-20{ }^{\circ} \mathrm{C}$ and reconstituted with distilled sterile water to the original volume before use. The optimal amount of bacteria used for LDT was empirically obtained as described below.

\section{Media evaluation test}

Before exposing $H$. contortus eggs and hatched larvae to fungal lectins, the effect of different culture conditions on larval vitality and development to L3 stage has been tested. Basic cultivation media contained $20 \mu \mathrm{l}$ yeast extract in Earle's balanced salt solution, prepared as described above, to which 1-6 $\mu$ l of either sterile water, sheep faecal extract as described in [36], living bacteria (E. coli OP50 cultivated overnight at $37{ }^{\circ} \mathrm{C}$ in LB media), or heat treated and lyophilised bacteria (prepared as above) were added. To each well, $10 \mu \mathrm{l}$ of egg solution containing approximately 50-60 eggs and 114-119 $\mu \mathrm{l}$ of distilled water was added to make a total volume of $150 \mu \mathrm{l}$ per well. Cultivation was done in 96-well flatbottom plates (TPP, Trasadingen, Switzerland) at $26{ }^{\circ} \mathrm{C}$ until day 7 , when the percentage of larvae developed to L3 was visually determined by light microscopy.

\section{Lectin production, purification and biotin labelling}

All lectins were produced and purified as described previously [19, 22-25, 32]. Briefly, corresponding cDNAs had been amplified and cloned in Escherichia coli strain DH5 $\alpha$ and the proteins were expressed in E. coli strain BL21 (DE3). The lectins were purified either via carbohydrate affinity chromatography using appropriate carbohydrate ligands, or via polyhistidine tag (6x His) using immobilized metal-affinity chromatography (IMAC), subsequently desalted on a PD-10 column (Amersham Biosciences) and concentrated using an Amicon Ultra-4 centrifugal filter device (Millipore) with a molecular weight cut-off of $10 \mathrm{kDa}$. For fluorescence microscopy, purified lectins were labelled with EZ-Link sulfo-NHS-biotin kit (Pierce) according to manufacturer's instructions, followed by desalting and concentration as for unlabelled lectins.

\section{Biotoxicity assay and statistical evaluation}

Media composition and volumes were based on the media evaluation test (see above). Each well contained $103 \mu \mathrm{l}$ of distilled water, $20 \mu \mathrm{l}$ yeast extract in Earle's balanced salt solution, $2 \mu \mathrm{l}$ heat-treated and lyophilized bacteria as larval food source, $10 \mu \mathrm{l}$ egg suspension with approximately $50 \mathrm{H}$. contortus eggs and either $15 \mu \mathrm{l}$ of 
lectin solutions or sterile water as negative control. Each sample was tested in triplicate and the assay was performed three times. CGL2, AAL and MOA lectins were tested at final concentrations of 1,5 and $10 \mu \mathrm{g} / \mathrm{ml}$, CCL2 was tested at 10, 20 and $40 \mu \mathrm{g} / \mathrm{ml}$, Tectonin and CGL3 were tested at concentrations of 10,50 and $100 \mu \mathrm{g} / \mathrm{ml}$. The concentrations were chosen on the base of preliminary toxicity assays. The plate was incubated at $26{ }^{\circ} \mathrm{C}$ and relative humidity of $100 \%$ for 7 days and checked daily for hatching of the eggs and phenotypic assessment of the larval development under a light microscope. After 7 days the number of larvae developed to L3 stage was determined and expressed as percentage relative to the control which was set as $100 \%$ development for the respective test.

The statistical significance of the toxicity assays was evaluated using a $t$-test for pairwise comparisons between each lectin test concentration and the untreated control.

\section{Fluorescence microscopy using biotinylated lectins Sample preparation}

Exposure of larvae to biotinylated lectins was performed in 24-well plates (TPP, Trasadingen, Switzerland) in a final volume of $1 \mathrm{ml}$. For this experiment all biotinylated lectins were tested at final concentration of $20 \mu \mathrm{g} / \mathrm{ml}$. Eggs were allowed to hatch overnight and L1 were further incubated for $9 \mathrm{~h}$ without change of media allowing ingestion of biotinylated lectins. After that, the lectincontaining medium was replaced with $0.85 \% \mathrm{NaCl}$ solution and larvae were allowed to empty their gut contents during the following $24 \mathrm{~h}$ with two more media exchanges $(0.85 \% \mathrm{NaCl})$ at $8 \mathrm{~h}$ intervals. Finally, the larvae were fixed and prepared for histochemistry as described below for adult specimens, except for the lectin incubation step.

Adults of Haemonchus contortus were obtained from the abomasum of experimentally infected sheep. The worms were washed 3 times in PBS and then fixed in 4. \% formaldehyde in PBS overnight at $4{ }^{\circ} \mathrm{C}$. Following fixation, the samples were rinsed once with PBS and incubated in $30 \%$ sucrose solution at $4{ }^{\circ} \mathrm{C}$ overnight (until they sank to the bottom of the tube). Subsequently, the sucrose solution was completely removed and the samples were embedded in O.C.T. medium (CellPath Ltd, UK) in plastic moulds and frozen on top of dry ice. The blocks were stored at $-80{ }^{\circ} \mathrm{C}$ until cutting (Cryostat 2800 Frigocout, Cambridge Instruments $\mathrm{GmbH}$ ). Sections of $\sim 7 \mu \mathrm{m}$ were air dried and further processed for fluorescence microscopy.

\section{Lectin histochemistry and microscopy}

The sections were rehydrated in PBS and additionally fixed with $4 \%$ formaldehyde $40 \mathrm{~min}$ at room temperature.
The sections were then washed with PBS and blocked in $0.1 \mathrm{M}$ glycine for $20 \mathrm{~min}$ at room temperature and further incubated in $2 \% \mathrm{BSA} / \mathrm{PBS}$ at $4{ }^{\circ} \mathrm{C}$ overnight. Following blocking step, the adult worm sections were probed with biotinylated lectins in $2 \% \mathrm{BSA} / \mathrm{PBS}$ at 5,10 and $20 \mu \mathrm{g} / \mathrm{ml}$ for $45 \mathrm{~min}$ at room temperature, washed three times with $1 \% \mathrm{BSA} / \mathrm{PBS}$ and incubated in $15 \mu \mathrm{g} / \mathrm{ml}$ Atto 655 Streptavidin (Sigma-Aldrich) in $2 \% \mathrm{BSA} / \mathrm{PBS}$ for another $45 \mathrm{~min}$ at room temperature. Finally, the samples were washed 4 times with $1 \%$ BSA/PBS (third wash contained $1 \mu \mathrm{g} / \mathrm{ml}$ DAPI, to stain nuclear DNA) embedded in mowiol or Vectashield (Vector Laboratories, Burlingame, CA, USA) and visualized using a Leica SP2 AOBS confocal laser scanning microscope (Leica Microsystems, Wetzlar, Germany). The acquisition settings (laser power, gain and offset) were determined on the control slide (probed with Atto 655 Streptavidin alone) by defining the signal threshold and then, without changing any of the acquisition parameters, the images were acquired from slides probed with biotinylated lectins.

The fluorescent channels were false-coloured using Zeiss LSM Image Browser and exported to CorelDRAW 12 software for preparation of the figures.

\section{Light microscopy of $H$. contortus larvae}

Differential interference contrast (DIC) images were acquired on a Leica DMI 6000B epifluorescent microscope using a 10x/0.30 inverted objective. Image acquisition and processing was done with Leica Application Suite AF software and exported to CorelDRAW 12 for preparation of the figures.

\section{Results}

\section{Media evaluation for larval cultivation}

To prevent commonly reported problems associated with contamination of larval cultures with unknown bacteria or fungi we aimed at a cleaner egg preparation. For this purpose, the standard egg sedimentation-flotation protocol was modified to include separation of eggs over the Percoll density gradient and extensive washing of eggs in antibiotic-antimycotic solution prior to seeding in the test plates. Furthermore, different culture media compositions were compared on their suitability to promote larval development to L3 stage. Of four different media containing different sources of food: yeast extract alone (Fig. 1a), yeast extract supplemented with sheep faecal extract (Fig. 1b), and yeast extract supplemented with either living (Fig. 1c) or heat-treated and lyophilized bacterial culture of E. coli OP50 strain (Fig. 1d), only the latter resulted in obtaining a reproducibly high percentage of $H$. contortus larvae that developed to L3 stage within one week of in vitro cultivation without inducing problems associated with bacterial or fungal contamination. The improved LDT resulted in egg hatching rate of $>95 \%$ 

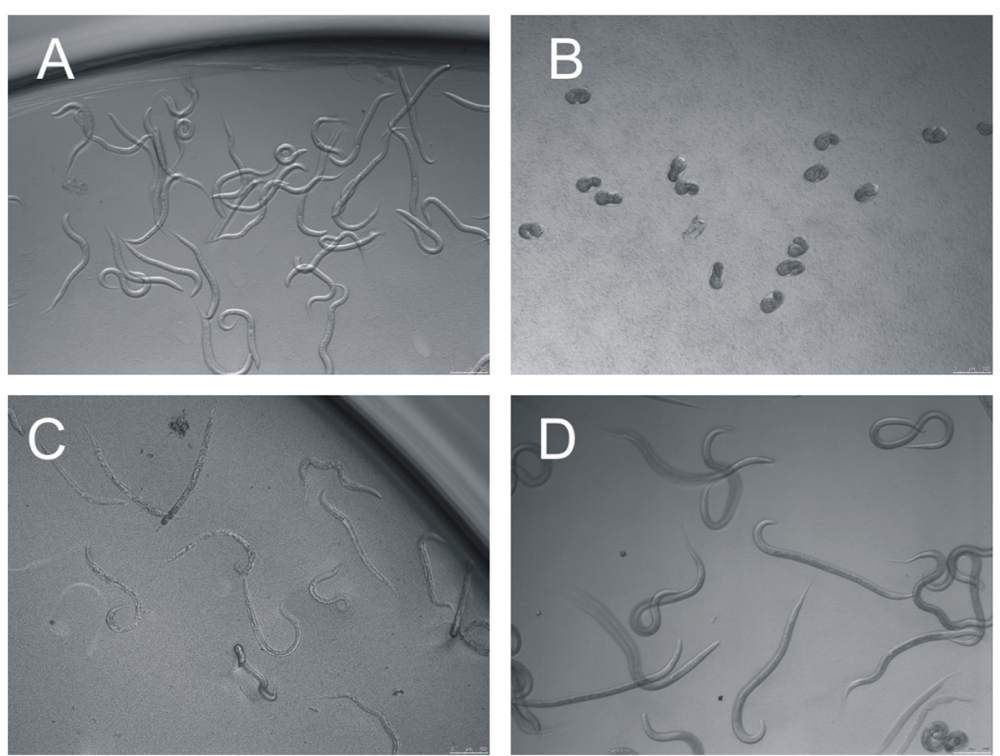

Fig. 1 Effect of different nutritive media on $\mathrm{H}$. contortus larval development. Basic medium containing only yeast extract and salts fails to promote larval development and the larvae stagnate in L1 stage (a). When sheep faecal extract from egg isolation (flow-through during sieving) is used as media supplement, none of the larvae hatch. The eggs embryonate, but the larvae die before hatching (b). Using living bacteria solution as a food source eventually leads to bacterial overgrowth during the cultivation period of 7 days resulting in death of the larvae and irreproducible $L 3$ counts (c). Addition of heat-treated and lyophilized bacteria solution supports development of larvae to L3 stage without inducing bacterial or fungal contamination (d)

and development of larvae to L3 stages of $\sim 75 \%$ in average of 18 experiments performed in triplicates (range 57.57 - $88.75 \%$, Additional file 1: Figure S1). Based on these results we used $2.0 \mu \mathrm{l}$ of heat-treated and lyophilized bacteria solution as food source in the lectin toxicity assay.

\section{Biotoxicity assay}

A set of six fungal lectins was tested in a biotoxicity assay for their potential to inhibit development of $H$. contortus larval stages. Tectonin and CGL3 had no visible effect at the tested concentrations, whereas AAL, CGL2, MOA and CCL2, demonstrated an inhibitory effect on the development of $H$. contortus larvae to L3 stage (Fig. 2). The highest potency was observed in larvae exposed to CGL2, AAL and MOA, in which case lectin concentrations of only $1 \mu \mathrm{g} / \mathrm{ml}$ strongly inhibited larval development (CGL2 $=72 \% p=0.016, \mathrm{AAL}=88 \%$ $p=0.004$, and $\mathrm{MOA}=50 \%$ inhibition $p=0.014$ ), while these three lectins in concentrations of $5 \mu \mathrm{g} / \mathrm{ml}$ almost completely ( $>98 \%$ ) prevented development to L3 stage. A dose-dependent inhibition of larval development was also observed in larvae exposed to CCL2 lectin, albeit at higher concentrations (e.g. $40 \mu \mathrm{g} / \mathrm{ml}$ to achieve $95 \%$ inhibition, $p<0.001$, Fig. 2). In the case of Tectonin and CGL3 lectins, exposure to concentrations as high as $100 \mu \mathrm{g} / \mathrm{ml}$ did not affect larval development during the test $(p>0.05)$. Regarding the effect of lectins on larval morphology, stunted larval growth (arrest in L1 stage) was observed in AAL, CCL2 and CGL2 lectins, without other visible alterations of phenotype or viability during one week cultivation. In contrast, the larvae exposed to MOA lectin displayed severe malformations in body shape, characterized predominantly by shrunk body appearance with undulating cuticle (Additional file 2: Figure S2). These morphological changes started being noticeable from day 4 and quickly led to death of affected larvae. The larvae exposed to Tectonin and CGL3 didn't show any phenotypic alterations compared to the control.

\section{Localisation of lectin binding sites in Haemonchus contortus}

In order to determine if biotoxicity correlates with the ability of lectins to bind glycan structures present in the worm gut, indirect immunofluorescence microscopy was performed on in situ labelled L1 stages and on fixed sections of adults using biotinylated lectins. Larvae which were exposed to the inhibitory lectins: MOA, AAL, CCL2 and CGL2, demonstrated lectin binding to the digestive tract distal from the oesophagus (Fig. 3, leftmost column). Additionally, the CCL2 lectin bound to the pharyngeal region of the L1 stages. Larvae incubated with the non-toxic lectins, Tectonin and CGL3 or with 


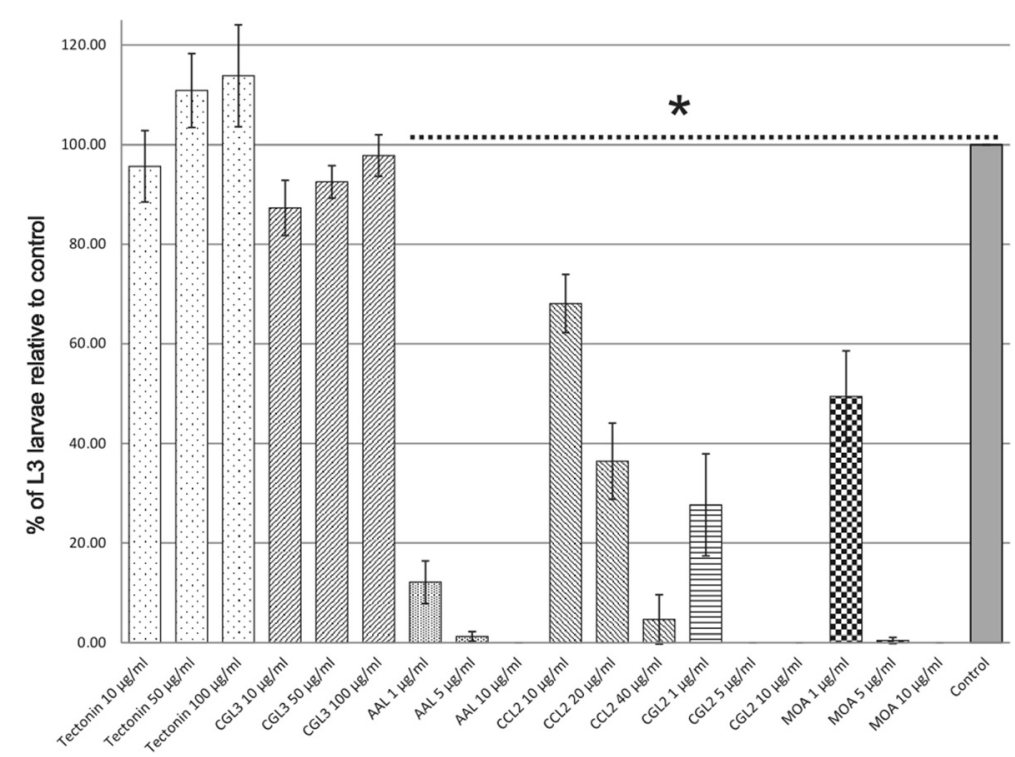

Fig. 2 Inhibitory effect of fungal lectins on the development of $H$. contortus larval stages. The graph shows the effect of exposure of $H$. contortus L1 larvae to six fungal lectins: Tectonin, CGL3, AAL, CCL2, CGL2, and MOA. Development of H. contortus larvae to L3 stage was quantified relative to respective untreated control which was set as $100 \%$. CCL2, CGL2, AAL, and MOA inhibited development of the larvae to L3 stage in a dosedependent manner. Bars represent the means of three independent experiments. Error bars indicate the standard deviations. Asterisk indicates statistically significant difference $(p<0.01)$

Atto 655 Streptavidin alone (negative control), did not show any fluorescent signal (Fig. 3, CGL3 not shown).

Because of practical issues, in situ labelling of living $H$. contortus adults with biotynilated lectins could not be performed. In order to determine if the glycan epitopes targeted by toxic lectins in L1 larvae are also found in the gastrodermal tissue of adult worms, formaldehyde fixed semi-thin sections of adult worms were probed with biotinylated lectins. In accordance with the results of in situ-labelled L1 stages, all four lectins that bound the gut of L1, also labelled the brush border of the adult gut (MOA, AAL, CCL2, CGL2; Fig. 3, $3^{\text {rd }}$ column). However, this method of sample preparation exposed worm tissues and glycan structures which most likely would not be accessible to the lectins in living adults. For example, fasciae of internal organs in the case of AAL and one of the inner cuticle layers in the case of MOA lectin also stained positive.

In contrast to toxic lectins, probing adult tissue sections with Tectonin (Fig. 3) and CGL3 lectin (data not shown) did not result in visible staining, supporting the results obtained with in situ-labelled L1 stages.

\section{Discussion}

Of the 4 tested cultivation media, addition of heattreated and lyophilized E. coli was the most suitable food source for supporting $H$. contortus larval development. The basic medium containing only yeast extract and salts, originally developed for the purpose of anthelmintic screening [37], failed to promote larval development during a one-week period. The larvae hatched, but stagnated in L1 stage (Fig. 1a). Other researchers circumvented this problem by adding bacteria isolated from sheep faecal extract [36]. Despite promoting larval development, assays showed high variability depending on the amount of faecal water used due to the non-standardised composition of microflora from different sources [38]. Furthermore, toxicity assays using lectins might fail because of possible interaction of lectins with ingredients of faeces from plant origin. In our assay, addition of faecal water resulted in complete inhibition of larval hatching (Fig. 1b) which can likely be associated with extreme changes in physical conditions caused by bacterial overgrowth and accumulation of metabolic waste products. Therefore, LDT that uses faecal extract is difficult to standardise and is not regarded suitable for lectin biotoxicity assays. The same authors describe the use of axenic culture of living E. coli bacteria as a food source for $H$. contortus larvae [36]. Despite improved larval development, the use of live bacteria frequently caused loss of experimental replicates due to bacterial overgrowth (Fig. 1c). In contrast, the addition of heat-treated and lyophilized $E$. coli to the basic medium, as described by Coles and colleagues [39], was sufficient to promote in vitro development of larvae to L3 stage (Fig. 1d), if problems associated with bacterial or fungal contamination 


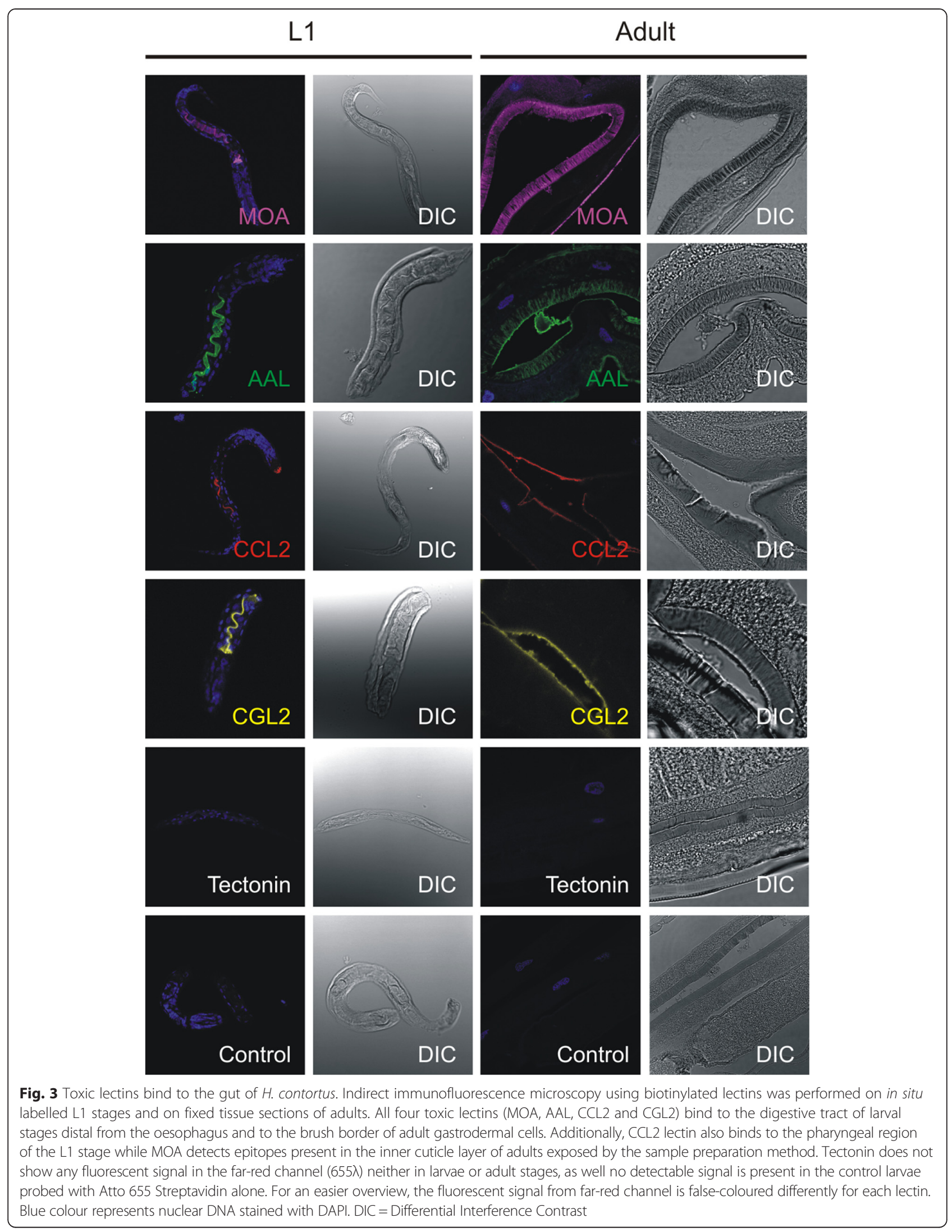


were avoided. In our experiments, this issue was alleviated by improved egg purification using Percoll gradient and subsequent extensive washes of eggs in antibioticantimycotic solution before seeding them in the test plate. This procedure resulted in larval development to L3 of $\sim 75 \%$ in average (Additional file 1: Figure S1) and supported one week cultivation of hatched larvae without inducing bacterial or fungal contamination (Fig. 1d).

Nematotoxic properties of the fungal fruiting body lectins used in our study were previously investigated in the free-living model nematode C. elegans. Five of the tested lectins, AAL, CCL2, MOA, CGL2, and Tectonin, demonstrated toxicity against larval stages of the nematode manifesting in inhibition of larval development, which in some cases (MOA, CCL2 and CGL2) resulted in worm death $[19,22-25,30,40]$. CGL3 lectin had no effect on nematode, insect and protozoan model organisms [24]. Conservation of the target glycan epitopes between C. elegans and the parasitic nematodes [21, 27, 40-42] and their location on the intestinal epithelium in C. elegans, led us to the hypothesis that the same lectins which are toxic to C. elegans might exhibit toxicity to $H$. contortus larval stages and therefore might serve as leads in discovery of novel hidden vaccine candidates. Actually, four of the five lectins toxic to C. elegans larvae, AAL, CCL2, MOA, and CGL2 were able to inhibit the development of $H$. contortus larvae in vitro in a dosedependent manner (Fig. 2). However, during one week cultivation only the larvae exposed to MOA lectin died as a result of the treatment, while the other three lectins AAL, CCL2 and CGL2 had a larvistatic effect that manifested as L1 stage arrest (Additional file 2: Figure S2). The results of the lectin toxicity assay on $H$. contortus larvae were in accordance with the data obtained by immunohistochemistry using biotinylated lectins. The gastrodermal tissue of both, in situ-labelled larvae and fixed adult sections, stained positive with lectins exhibiting toxicity, while the two lectins which had no effect on the development of larvae, Tectonin and CGL3, were negative (Fig. 2, CGL3 not shown). CGL3 lectin was shown to specifically bind to LacdiNAc (GalNAc-beta1,4-GlcNAc) which was found on $\mathrm{N}$-glycan antenna of insects [43] and recently also in $H$. contortus [26], but exposure of larvae to this lectin was interestingly not toxic. This epitope, in fucosylated and non-fucosylated form, is discussed as a vaccine candidate against schistosomes because it was shown to contribute to the ability of rhesus monkeys to clear the infection [44]. Intriguingly Tectonin, which was toxic to C. elegans larvae, had no effect on development of $H$. contortus larvae. Tectonin target are O-methylated mannose and fucose residues on $\mathrm{N}$-glycan antenna in $C$. elegans and it was shown that a functional samt-1 gene, possibly coding for a Golgi SAM-transporter, is required for their biosynthesis [25]. Although a C. elegans samt-1 homolog is present in the genome of $H$. contortus $(P=2.6 \mathrm{e}-164)$ to date there have been no reports of $\mathrm{O}$-methylated glycans in this parasitic nematode. It is possible that in $H$. contortus, the glycans targeted by CGL3 and Tectonin have a temporal (during larval development) or spatial distribution that prevents them to be targeted by orally ingested lectins. An example of different spatial distribution of conserved glycans between free-living and parasitic nematodes is found in Toxocara canis [45] where methylated glycans constitute a part of excretory/secretory antigen, while in C. elegans they localize on the gut epithelium and body surface [25]. Based on phenotypic assessment of larval stages and localization of lectin binding sites, we can assume that there is a difference in the mode of toxicity between the lectins. CGL2, CCL2 and AAL recognised epitopes on the apical surface of the gut microvilli of the adults and exposure of larvae to those lectins manifested in growth arrest without killing the larvae during one week of cultivation. The observed phenotype closely resembles the effect of cultivating the larvae in basal media containing no bacteria as food source (Fig. 1a). It is important to note that the function of pharynx seems to be unaffected, as judged by the ability of biotinylated lectins to bind the gut after pharyngeal bulb. Therefore, it is possible that binding of CGL2, CCL2 and AAL lectins to the luminal surface of the gut microvilli interferes with resorption of nutrients in larval stages. These results are in accordance with our recent results on the nematotoxicity of CCL2 in C. elegans [30]. In contrast, the larvae exposed to MOA lectin displayed characteristic deformations in morphology during development, manifesting in shrunk body and visibly enlarged intestine after pharynx/ bulbus (Additional file 2: Figure S2) and died before reaching the L3 stage. Observed structural deformations of the gut closely resemble those previously described in a C. elegans study which demonstrated that MOA-mediated nematotoxicity is directly linked to binding of the lectin to glycosphingolipids of the worm and further biochemical characterization revealed a critical role of cysteine protease activity in this process [19]. The vaccine potential of the glycoepitope targeted by MOA lectin is further confirmed by a study that examined the sera of protected lambs using glycan microarray analysis in which two glycan antigens that possibly contribute to protection against $H$. contortus challenge infection were identified [18]. One of those, the Galo1-3GalNac, is the glycan targeted by MOA lectin. Combining toxicity of MOA to larval stages, protective antibodies in the lamb serum against MOA target glycan and immunohistochemistry studies confirming gut localisation of this epitope in adult worms (and therefore accessibility of the epitope to the specific protective antibodies) it is realistic to conclude that the Galo13 GalNac epitope presents a promising vaccine candidate 
against $H$. contortus. Toxicity of CGL2 towards C. elegans was shown to be mediated by binding of the lectin to the Gal- $\beta 1,4$-Fuc- $\alpha 1,6$-epitope (Gal-Fuc epitope) on the proximal GlcNAc residue of $\mathrm{N}$-glycan cores [22]. The Gal-Fuc epitope has been identified in parasitic nematodes Ascaris suum and Oesophagostomum dentatum [41] and recently, the existence of this and yet another Gal-Fuc epitope have been demonstrated for cores of $H$. contortus N-glycans [26].

Similarly, CCL2 lectin targets $\alpha 1,3$ fucosylated N-glycan cores [23] which have been previously identified as antigens in $H$. contortus [27]. Interestingly, one of the most promising $H$. contortus vaccine candidates, the native aminopeptidase $\mathrm{H} 11$ expressed in the worm gut has been shown to contain this glycan modification [29]. Since other expression systems failed to stimulate comparable levels of protection to the native antigen, attempts have been made to produce correctly folded and glycosylated $\mathrm{H} 11$ by using C. elegans as expression system [28]. This approach, however, did not reduce the $H$. contortus worm burden or egg shedding, possibly because the glycan structures made by C. elegans were not completely identical to those of $H$. contortus, or there are additional factors involved in the protective effect of the native $\mathrm{H} 11$ antigen preparation. In this regard, the conservation of glycoepitopes between different nematode species might offer the possibility to use a non-parasitic, free-living species as a source of the antigen for a first generation of vaccines.

Little is known about the glycan targets of the AAL lectin in H. contortus and C. elegans. Genetic data suggests that the core fucose-residues on $\mathrm{C}$. elegans $\mathrm{N}$-glycan are not responsible for the nematotoxicity of this fucosebinding lectin [24]. Alternative AAL targets are fucosylated O-glycans which have recently been detected in C. elegans [46]. Nothing is known about the structure of $\mathrm{O}$-glycans in $H$. contortus to date.

\section{Conclusions}

In addition to what we know from studies in free-living nematodes, the in vitro studies reported here provide novel data regarding the effects of nematotoxic fungal lectins against $H$. contortus larval stages. We show that effector lectins of a fungal defence system targeting conserved glycans in predatory nematodes in nature, may serve as a tool for the identification of potential targets of the vertebrate immune system in parasitic nematodes. The spatial and developmental distribution of the target glycans of the fungal lectins in $H$. contortus reveals them as hypothetical hidden antigens and thus further supports this idea. The question whether these epitopes can be used as effective vaccines against parasitic nematodes, however, is still open and has to be experimentally validated.

\section{Additional files}

Additional file 1: Figure S1. Standardization of the larval development assay. In eighteen independent experiments performed in triplicates under conditions of egg preparation and in vitro cultivation of larvae as described in the material and methods section, in average $\sim 75 \%$ of larvae developed to L3 stages during one week cultivation (range $57.57 \%-88.75 \%$ ). (TIFF $2385 \mathrm{~kb}$ )

Additional file 2: Figure S2. Phenotypic changes in $\mathrm{H}$. contortus larvae exposed to toxic lectins in vitro. Comparison of phenotypic manifestations in $\mathrm{H}$. contortus larvae exposed to lectins during one week in vitro cultivation. In the low concentration of MOA lectin $(1 \mu \mathrm{g} / \mathrm{ml})$ about $50 \%$ larvae develop to $L 3$ stage, while the affected larvae are shorter and display irregular body morphology. Exposure to higher concentrations of MOA $(5 \mu \mathrm{g} / \mathrm{ml})$ results in death of most of the larvae ( $\geq 98 \%$, note shrunk cuticles and irregular body shapes). Larvistatic effect can be observed in larvae exposed to CCL2, CGL2 and AAL lectins. The larvae survive, but fail to develop to L3 stage (note the difference in size of one larva representing $L 3$ stage compared to the other larvae which are arrested in L1 stage in AAL sample). All images were acquired using the same optical magnification (10x) and size differences are to the scale. Scale bars $=200 \mu \mathrm{m}$. (TIFF $2309 \mathrm{~kb}$ )

\section{Competing interests}

The authors declare that they have no competing interests.

\section{Authors' contributions}

$\mathrm{HH}, \mathrm{MA}, \mathrm{PD}, \mathrm{MK}$, and SS conceived and designed the study. $\mathrm{CH}, \mathrm{AB}, \mathrm{SBM}$ and SS carried out experimental work ( $\mathrm{CH}$ and SS performed lectin toxicity assays, histochemistry and microscopy, $A B$ and SBM performed lectin production, purification and labelling). $\mathrm{CH}, \mathrm{HH}, \mathrm{AB}, \mathrm{SBM}, \mathrm{MA}, \mathrm{PD}$ MK and SS wrote the manuscript or significantly contributed in intellectual content and data analysis. All authors read and approved the final manuscript.

\section{Acknowledgements}

The project was funded by the "Kommision für Technologie und Innovation KTI" research grant F-52201-22-01 and provided support to C.H. and in part to S.S.

We would like to thank the people of Malcisbo AG, a ETH startup company for their continuing help and support. Special thank goes to Bruno Oesch for critically reading the manuscript. We would also like to acknowledge Hanspeter Müller for his knowledgeable assistance in animal experimentation.

\section{Author details}

${ }^{1}$ Institute of Parasitology, University of Zurich, Winterthurerstrasse 266a, 8057 Zurich, Switzerland. ${ }^{2}$ Malcisbo AG, Wagistrasse 27a, 8952 Schlieren, Switzerland. ${ }^{3}$ Institute of Microbiology, Swiss Federal Institute of Technology (ETH) Zürich, 8093 Zürich, Switzerland.

Received: 13 April 2015 Accepted: 5 August 2015

Published online: 19 August 2015

\section{References}

1. Besier RB, Love SCJ. Anthelmintic resistance in sheep nematodes in Australia: the need for new approaches. Aust J Exp Agric. 2003;43(12):1383-91.

2. Papadopoulos E. Anthelmintic resistance in sheep nematodes. Small Ruminant Res. 2008;76(1-2):99-103.

3. Van den Brom R, Moll L, Borgsteede FH, Van Doorn DC, Lievaart-Peterson K, Dercksen DP, et al. Multiple anthelmintic resistance of Haemonchus contortus, including a case of moxidectin resistance, in a Dutch sheep flock. Vet Rec. 2013;173(22):552.

4. Mederos AE, Banchero GE, Ramos Z. First report of monepantel Haemonchus contortus resistance on sheep farms in Uruguay. Parasites Vectors. 2014;7(1):598.

5. Almeida FA, Garcia KC, Torgerson PR, Amarante AF. Multiple resistance to anthelmintics by Haemonchus contortus and Trichostrongylus colubriformis in sheep in Brazil. Parasitol Int. 2010;59(4):622-5.

6. Jacobs HJ, Wiltshire C, Ashman K, Meeusen ENT. Vaccination against the gastrointestinal nematode, Haemonchus contortus, using a purified larval surface antigen. Vaccine. 1999;17(4):362-8. 
7. Schallig HDFH, van Leeuwen MAW. Protective immunity to the bloodfeeding nematode Haemonchus contortus induced by vaccination with parasite low molecular weight antigens. Parasitology. 1997;114(03):293-9.

8. Schallig HDFH, Van Leeuwen MAW, Cornelissen AWCA. Protective immunity induced by vaccination with two Haemonchus contortus excretory secretory proteins in sheep. Parasite Immunol. 1997:19(10):447-53.

9. Smith WD, Smith SK, Murray JM. Protection studies with integral membrane fractions of Haemonchus contortus. Parasite Immunol. 1994;16(5):231-41.

10. Smith SK, Smith WD. Immunisation of sheep with an integral membrane glycoprotein complex of Haemonchus contortus and with its major polypeptide components. Res Vet Sci. 1996;60(1):1-6.

11. Newton SE, Munn EA. The Development of Vaccines against Gastrointestinal Nematode Parasites, Particularly Haemonchus contortus. Parasitol Today. 1999;15(3):116-22.

12. Knox DP, Redmond DL, Newlands GF, Skuce PJ, Pettit D, Smith WD. The nature and prospects for gut membrane proteins as vaccine candidates for Haemonchus contortus and other ruminant trichostrongyloids. Int J Parasitol. 2003;33(11):1129-37.

13. Cachat E, Newlands GFJ, Ekoja SE, McAllister H, Smith WD. Attempts to immunize sheep against Haemonchus contortus using a cocktail of recombinant proteases derived from the protective antigen, $\mathrm{H}$-gal-GP. Parasite Immunol. 2010;32(6):414-9.

14. Newton SE, Meeusen ENT. Progress and new technologies for developing vaccines against gastrointestinal nematode parasites of sheep. Parasite Immunol. 2003;25(5):283-96.

15. Vervelde L, Van Leeuwen MA, Kruidenier M, Kooyman FN, Huntley JF, van Die l, et al. Protection studies with recombinant excretory/secretory proteins of Haemonchus contortus. Parasite Immunol. 2002;24(4):189-201.

16. Smith TS, Graham M, Munn EA, Newton SE, Knox DP, Coadwell WJ, et al. Cloning and characterization of a microsomal aminopeptidase from the intestine of the nematode Haemonchus contortus. Biochim Biophys Acta. 1997;1338(2):295-306.

17. Geldhof P, Newlands GF, Nyame K, Cummings R, Smith WD, Knox DP. Presence of the LDNF glycan on the host-protective H-gal-GP fraction from Haemonchus contortus. Parasite Immunol. 2005;27(1-2):55-60

18. van Stijn CMW, van den Broek M, Vervelde $L$, Alvarez RA, Cummings RD, Tefsen B, et al. Vaccination-induced lgG response to Gala1-3GalNAc glycan epitopes in lambs protected against Haemonchus contortus challenge infection. Int J Parasitol. 2010;40(2):215-22

19. Wohlschlager T, Butschi A, Zurfluh K, Vonesch SC, auf dem Keller U, Gehrig $P$, et al. Nematotoxicity of Marasmius oreades agglutinin (MOA) depends on glycolipid binding and cysteine protease activity. J Biol Chem. 2011;286(35):30337-43

20. Singh RS, Bhari R, Kaur HP. Mushroom lectins: current status and future perspectives. Crit Rev Biotechnol. 2010;30(2):99-126.

21. Kunzler M. Hitting the sweet spot-glycans as targets of fungal defense effector proteins. Molecules. 2015;20(5):8144-67.

22. Butschi A, Titz A, Walti MA, Olieric V, Paschinger $K$, Nobauer $K$, et al. Caenorhabditis elegans $\mathrm{N}$-glycan core beta-galactoside confers sensitivity towards nematotoxic fungal galectin CGL2. PLoS Pathog. 2010;6(1):e1000717.

23. Schubert M, Bleuler-Martinez S, Butschi A, Walti MA, Egloff $P$, Stutz $K$, et al. Plasticity of the beta-trefoil protein fold in the recognition and control of invertebrate predators and parasites by a fungal defence system. PLoS Pathog. 2012;8(5);e1002706.

24. Bleuler-Martinez S, Butschi A, Garbani M, Walti MA, Wohlschlager T, Potthoff E, et al. A lectin-mediated resistance of higher fungi against predators and parasites. Mol Ecol. 2011;20(14):3056-70

25. Wohlschlager T, Butschi A, Grassi P, Sutov G, Gauss R, Hauck D, et al. Methylated glycans as conserved targets of animal and fungal innate defense. Proc Natl Acad Sci U S A. 2014;111(27):e2787-96.

26. Paschinger $\mathrm{K}$, Wilson IB. Two types of galactosylated fucose motifs are present on N-glycans of Haemonchus contortus. Glycobiology. 2015;25(6):585-90.

27. van Die I, Gomord V, Kooyman FN, van den Berg TK, Cummings RD, Vervelde L. Core alpha1 $\rightarrow 3$-fucose is a common modification of $\mathrm{N}$-glycans in parasitic helminths and constitutes an important epitope for lgE from Haemonchus contortus infected sheep. FEBS Lett. 1999;463(1-2):189-93.

28. Roberts B, Antonopoulos A, Haslam SM, Dicker AJ, McNeilly TN, Johnston SL, et al. Novel expression of Haemonchus contortus vaccine candidate aminopeptidase $\mathrm{H} 11$ using the free-living nematode Caenorhabditis elegans. Vet Res. 2013;44:111.

29. Haslam SM, Coles GC, Munn EA, Smith TS, Smith HF, Morris HR, et al. Haemonchus contortus glycoproteins contain N-linked oligosaccharides with novel highly fucosylated core structures. J Biol Chem. 1996;271(48):30561-70

30. Stutz K, Kaech A, Aebi M, Kunzler M, Hengartner MO. Disruption of the C. elegans Intestinal Brush Border by the Fungal Lectin CCL2 Phenocopies Dietary Lectin Toxicity in Mammals. PLoS One 2015, 10(6):e0129381.

31. Walser PJ, Haebel PW, Kunzler M, Sargent D, Kues U, Aebi M, et al. Structure and functional analysis of the fungal galectin CGL2. Structure 2004, 12(4):689-702

32. Walti MA, Walser PJ, Thore S, Grunler A, Bednar M, Kunzler M, et al. Structural basis for chitotetraose coordination by CGL3, a novel galectinrelated protein from Coprinopsis cinerea. Journal of molecular biology 2008, 379(1):146-159.

33. Fujihashi M, Peapus DH, Kamiya N, Nagata Y, Miki K. Crystal structure of fucose-specific lectin from Aleuria aurantia binding ligands at three of its five sugar recognition sites. Biochemistry 2003, 42(38):11093-99.

34. Wimmerova M, Mitchell E, Sanchez JF, Gautier C, Imberty A. Crystal structure of fungal lectin: six-bladed beta-propeller fold and novel fucose recognition mode for Aleuria aurantia lectin. J Biol Chem 2003, 278(29):27059-67.

35. Cordara G, Egge-Jacobsen W, Johansen HT, Winter HC, Goldstein IJ, Sandvig K, Krengel $\mathrm{U}$. Marasmius oreades agglutinin (MOA) is a chimerolectin with proteolytic activity. Biochemical and biophysical research communications 2011, 408(3):405-410

36. Hubert J, Kerboeuf D. A microlarval development assay for the detection of anthelmintic resistance in sheep nematodes. Vet Rec. 1992;130(20):442-6.

37. Taylor MA. A larval development test for the detection of anthelmintic resistance in nematodes of sheep. Res Vet Sci. 1990;49(2):198-202.

38. Taylor MA, Hunt KR, Goodyear KL. Anthelmintic resistance detection methods. Vet Parasitol. 2002;103(3):183-94.

39. Coles GC, Tritschler JP, 2nd, Giordano DJ, Laste NJ, Schmidt AL. Larval development test for detection of anthelmintic resistant nematodes. Res Vet Sci. 1988:45(1):50-3.

40 Kunzler M, Bleuler-Martinez S, Butschi A, Garbani M, Luthy P, Hengartner MO, et al. Biotoxicity assays for fruiting body lectins and other cytoplasmic proteins. Methods Enzymol. 2010;480:141-50.

41. Yan S, Bleuler-Martinez S, Plaza DF, Kunzler M, Aebi M, Joachim A, et al. Galactosylated fucose epitopes in nematodes: increased expression in a Caenorhabditis mutant associated with altered lectin sensitivity and occurrence in parasitic species. J Biol Chem. 2012;287(34):28276-90.

42. Wei JZ, Hale K, Carta L, Platzer E, Wong C, Fang SC, et al. Bacillus thuringiensis crystal proteins that target nematodes. Proc Natl Acad Sci U S A. 2003;100(5):2760-5.

43. Kurz S, Aoki K, Jin C, Karlsson NG, Tiemeyer M, Wilson IB, et al. Targeted release and fractionation reveal glucuronylated and sulphated $\mathrm{N}$ - and O-glycans in larvae of dipteran insects. J Proteomics. 2015;126:172-88.

44. Luyai AE, Heimburg-Molinaro J, Prasanphanich NS, Mickum ML, Lasanajak Y, Song $X$, et al. Differential expression of anti-glycan antibodies in schistosome-infected humans, rhesus monkeys and mice. Glycobiology. 2014;24(7):602-18

45. Schabussova I, Amer H, van Die I, Kosma P, Maizels RM. O-methylated glycans from Toxocara are specific targets for antibody binding in human and animal infections. Int J Parasitol. 2007;37(1):97-109.

46. Palaima E, Leymarie N, Stroud D, Mizanur RM, Hodgkin J, Gravato-Nobre MJ, et al. The Caenorhabditis elegans bus-2 mutant reveals a new class of O-glycans affecting bacterial resistance. J Biol Chem. 2010;285(23):17662-72. 